\title{
Evidence that tRNA modifying enzymes are important in vivo targets for 5 -fluorouracil in yeast
}

\author{
MARIE GUSTAVSSON and HANS RONNE \\ Department of Medical Biochemistry and Microbiology, Uppsala University, SE-751 23 Uppsala, Sweden
}

\begin{abstract}
We have screened a collection of haploid yeast knockout strains for increased sensitivity to 5-fluorouracil (5-FU). A total of 138 5-FU sensitive strains were found. Mutants affecting rRNA and tRNA maturation were particularly sensitive to 5-FU, with the tRNA methylation mutant trm10 being the most sensitive mutant. This is intriguing since trm10, like many other tRNA modification mutants, lacks a phenotype under normal conditions. However, double mutants for nonessential tRNA modification enzymes are frequently temperature sensitive, due to destabilization of hypomodified tRNAs. We therefore tested if the sensitivity of our mutants to 5-FU is affected by the temperature. We found that the cytotoxic effect of 5-FU is strongly enhanced at $38^{\circ} \mathrm{C}$ for tRNA modification mutants. Furthermore, tRNA modification mutants show similar synthetic interactions for temperature sensitivity and sensitivity to 5-FU. A model is proposed for how 5-FU kills these mutants by reducing the number of tRNA modifications, thus destabilizing tRNA. Finally, we found that also wild-type cells are temperature sensitive at higher concentrations of 5-FU. This suggests that tRNA destabilization contributes to 5-FU cytotoxicity in wild-type cells and provides a possible explanation why hyperthermia can enhance the effect of 5 -FU in cancer therapy.
\end{abstract}

Keywords: 5-fluorouracil; pseudouridine synthase; tRNA methylase; tRNA modifications; yeast

\section{INTRODUCTION}

The pyrimidine analog 5-fluorouracil (5-FU) was developed more than 40 years ago and is still one of the most useful antitumor agents for treatment of solid cancers (Longley et al. 2003). In spite of this, its mechanism of action is still not fully understood. Thymidylate synthase (TS) is thought to be one important target of 5-FU (Parker and Cheng 1990; Longley et al. 2003). In mammalian cells, $5-\mathrm{FU}$ is converted to FdUMP, which forms a stable complex with TS, and thus inhibits dTMP production. dTMP is essential for DNA replication and repair, and its depletion therefore causes cytotoxicity. The mechanisms by which cytotoxicity is induced are not fully understood, but one important component is p53-dependent apoptosis (Longley et al. 2003).

However, inactivation of TS is not the only pathway for 5-FU cytotoxicity. Heimer and Sartorelli (1992) found that addition of exogenous uracil, but not thymidine, can suppress the cytotoxic effect of 5-FU, which suggests that 5-FU has other targets than TS. 5-FU can be converted into

Reprint requests to: Hans Ronne, Department of Medical Biochemistry and Microbiology, Uppsala University, Box 582, SE-751 23 Uppsala, Sweden; e-mail: Hans.Ronne@imbim.uu.se; fax: + 46-18-4714673.

Article published online ahead of print. Article and publication date are at http://www.rnajournal.org/cgi/doi/10.1261/rna.966208.
FdUTP and FUTP, which are incorporated into DNA and RNA, respectively. Interference with the biosynthesis or function of nucleic acids is therefore another possible mechanism of action for 5-FU. The results of two genomic screens in yeast suggested that rRNA maturation is an important target for 5-FU (Giaever et al. 2004; Lum et al. 2004). Both screens used haploinsuffiency profiling in yeast, in which heterozygous diploids are scored for increased sensitivity to a drug, and the majority of the genes found in the screens were involved in rRNA maturation. 5FU has also been shown to enhance exosome-dependent accumulation of polyadenylated rRNAs (Fang et al. 2004). Finally, there is recent evidence that 5-FU inhibits premRNA splicing, through its effect on pseudouridylation of U2 snRNA (Zhao and Yu 2007).

We have screened a haploid knockout strain collection in the yeast Saccharomyces cerevisiae for increased sensitivity to 5-FU. Such a screen is expected to reveal a different set of genes than a haploinsuffiency screen, since the effect of a complete absence of a protein is examined rather than the effect of a $50 \%$ reduction in its expression. A total of 138 knockout mutants that are hypersensitive to 5-FU were identified among 4676 mutants screened. Only four of the genes found in the two previous screens were identified in our screen. Instead, we found that several of the most sensitive mutants lack tRNA modification enzymes, thus identifying 
tRNA maturation as an important in vivo target for 5-FU. Interestingly, we further found that several tRNA modification mutants are temperature sensitive in the presence of 5-FU, and that genetic interactions between different tRNA modification mutants on $5-\mathrm{FU}$ mimic those seen at $38^{\circ} \mathrm{C}$.

Based on these findings, we propose that the combined effect of 5-FU and loss of a tRNA modifying enzyme causes destabilization of tRNA, with a resulting temperaturesensitive phenotype. Interestingly, we found that also wildtype cells are temperature sensitive in the presence of higher concentrations of 5-FU. This suggests that destabilization of tRNA contributes to 5-FU cytotoxicity in wild-type cells, which might explain why hyperthermia can enhance the effect of 5-FU in cancer therapy (Kouloulias et al. 2005).

\section{RESULTS}

\section{A genome-wide screen for haploid deletion strains sensitive to 5 -fluorouracil}

In a search for new targets of 5-FU, we screened 4676 haploid yeast deletion mutants for sensitivity to 5 -FU. We choose a 5 -FU concentration, $15 \mu \mathrm{g} / \mathrm{mL}$, which partially inhibits growth of the wild type in order to be able to find both resistant and sensitive mutants. However, only the latter were found in the screen. Sensitive deletion mutants were retested in both mating types. Of 212 strains initially scored as 5-FU sensitive, 74 were excluded since they were petites or had other general growth defects, which makes it more difficult to score drug sensitivity. The remaining 138 5-FU-sensitive strains are listed in Table 1; of these, 124 were deleted for verified genes, while 14 carried deletions of dubious open reading frames (ORFs) that are not conserved in closely related yeasts. Six of these ORFs overlap with verified genes that appeared in our screen, and we assume that the 5-FU-sensitive phenotype in these cases is due to loss of the verified gene. Three dubious ORFs overlap with essential genes. Since dubious ORFs are thought to lack functions (see www.yeastgenome.org), we consider it likely that it is the partial deletion of the essential gene that causes 5-FU sensitivity. The corresponding verified genes are shown in parenthesis in Table 1. Finally, five dubious ORFs overlap with verified nonessential genes that were included in our screen but did not score as 5-FU sensitive. A possible explanation in these cases would be if the partial deletion of the verified gene causes a truncated protein to be expressed, which has a dominant negative effect that increases the sensitivity to 5-FU. These genes are therefore also shown in parenthesis in Table 1.

\section{Functional classification of the genes}

Disregarding the six dubious ORF knockouts that overlap with verified 5-FU-sensitive genes, our screen yielded 132 unique genes, eight of which were only tentatively assigned as 5-FU sensitive by knockouts of overlapping dubious ORFs. Table 1 shows these 132 genes classified into functional groups. The largest functional group was genes involved in intracellular transport (24 genes). Such genes frequently appear in drug screens (Murén et al. 2001), probably because the uptake of drugs, and also their detoxification in the vacuole, is affected by defects in intracellular transport. We also found four genes involved in another membrane-related process, sterol biosynthesis. The reason why they appeared remains to be determined, but we note that a link exists between sterol biosynthesis and tRNA modification (Benko et al. 2000). Since genes involved in tRNA modification appeared prominently in our screen (see below), it is possible that this is why the sterol biosynthetic genes also were recovered.

The second largest group (23 genes) was those involved in transcription. This is also a prominent group in most genetic screens since transcription mutants may affect the process under study indirectly, through effects on the expression of genes that are directly involved in the process. Two genes encoding RNA polymerase I subunits (RPA34 and RPA49) were recovered, which is consistent with maturation and processing of rRNA being an important target for 5-FU (see below). Another functional group that appears in many genetic screens are genes involved in protein modification and degradation. We found nine genes belonging to this group. The third largest group (19 genes) were genes involved in DNA repair (Table 1). This was not unexpected since DNA damage, both indirectly, through inhibition of TS, and directly, through misincorporation of FdUTP into DNA, is though to be a major mechanism for 5-FU cytotoxicity. We note, however, that none of the most sensitive mutants belonged to this group.

We further found 10 genes involved in nuclear transport and 12 genes involved in rRNA maturation. Two of the most sensitive mutants in the screen were $\operatorname{rrp} 8$ and tom 1 (Table 1). Rrp8p is a highly conserved nucleolar protein and a putative methyltransferase (Antonelli-Bousquet et al. 2000). The E3 ubiquitin ligase Tom $1 p$ is a multifunctional nuclear protein required for the activity of the SAGA complex, mRNA export, and rRNA processing (Saleh et al. 1998; Duncan et al. 2000; Tabb et al. 2001). We also found 11 genes encoding ribosomal proteins or proteins involved in ribosome assembly, processes that are functionally linked to rRNA. This included the hit1 mutant, which was one of the most sensitive mutants in the screen. Hitlp interacts with Rsalp, which suggests that it is involved in ribosome assembly (Ito et al. 2001).

\section{tRNA modification mutants are highly sensitive to 5-fluorourcail}

Interestingly, we found that several of the most sensitive mutants are deleted for genes involved in tRNA modification, a process that has not previously been strongly linked to 
TABLE 1. Functional classification of genes recovered in the screen

\begin{tabular}{|c|c|c|}
\hline Functional class & Sensitivity & Gene \\
\hline \multicolumn{3}{|c|}{ DNA damage response } \\
\hline & +++ & DEF1, RAD17, RAD50, RAD51, TOP3 \\
\hline & ++ & $\begin{array}{l}\text { DDC1, HPR5, LRP1, MCK1, RAD24, RAD27, RAD52, RAD54, } \\
\text { RAD55, RMI1, UNG1, XRS2 }\end{array}$ \\
\hline & + & $M E C 3, R A D 6$ \\
\hline \multicolumn{3}{|c|}{ Ergosterol biosynthesis } \\
\hline & ++++ & ERG24 \\
\hline & +++ & ERG3, ERG6 \\
\hline & + & ERG4 \\
\hline \multicolumn{3}{|c|}{ Intracellular transport } \\
\hline & +++ & AKR1, ARC18, ARP5, END3, SHE1, VID21 \\
\hline & ++ & $\begin{array}{l}\text { ATG14, CDC50, GCS1, KCS1, SAC1, SLM4, SLM6, SRV2, VAM6, } \\
\quad \text { VPS41, VRP1 }\end{array}$ \\
\hline & + & EDE1, FPR2, LUV1, SAC6, TED1, VMA13, YPT7 \\
\hline \multicolumn{3}{|c|}{ Nucleocytoplasmic transport } \\
\hline & $+++(+)$ & TOM1 \\
\hline & +++ & LOS1, NUP170, PUS1 \\
\hline & ++ & DHH1, NPL3, SRP4O \\
\hline & + & MOG1, NUP188, (RLR1) \\
\hline \multicolumn{3}{|c|}{ Protein modification and degradation } \\
\hline & $+++(+)$ & TOM1 \\
\hline & +++ & DEF1, UBP15, VAN1 \\
\hline & ++ & OST4, UBX3 \\
\hline & + & (SEL1), UBA4, URM1 \\
\hline \multicolumn{3}{|c|}{ Ribosome subunits, assembly and function } \\
\hline & ++++ & HIT1 \\
\hline & +++ & ASC1, (RPL12B), RPL29, RSA1, TMA23 \\
\hline & ++ & RPL13B, RPL22A, RPL34B, (RPL43A), SRP40 \\
\hline \multicolumn{3}{|l|}{ rRNA processing } \\
\hline & $+++(+)$ & RRP8, TOM1 \\
\hline & +++ & LSM6, (NOP58) \\
\hline & ++ & DBP3, LRP1, NOP12, PIH1, REX4, RRP6 \\
\hline & + & NOP16, SSF1 \\
\hline \multicolumn{3}{|l|}{ Transcription } \\
\hline & +++ & ARG82, GCR2, IES6, REG1, RPA49, TAF14 \\
\hline & ++ & $\begin{array}{l}\text { BDF1, CAD1, GAL11, GCN5, IKI3, ISW1, (NET1), NGG1, RPN4, } \\
\text { SNF12, SSN3, STB5 }\end{array}$ \\
\hline & + & HTZ1, KTI12, (RLR1), RPA34, SSN2 \\
\hline \multicolumn{3}{|c|}{ tRNA modification } \\
\hline & ++++ & TRM10 \\
\hline & +++ & PUS1, TAN1, TRM1 \\
\hline & ++ & DUS3, IKI3, MOD5, TRM8, TRM82 \\
\hline & + & KTI12 \\
\hline \multicolumn{3}{|l|}{ Miscellaneous } \\
\hline & +++ & BEM1, REI1, SIS2 \\
\hline & ++ & CCS1, CKB2, (ERV2), GND1, GUP1, HSP31, KAR3, NBP2, RPE1 \\
\hline & + & (HRR25), ISC1, MET1, MRK1, URA5 \\
\hline \multicolumn{3}{|c|}{ Unknown function } \\
\hline & ++ & YNR068C \\
\hline & + & AlM9 \\
\hline \multicolumn{3}{|c|}{$\begin{array}{l}\text { Dubious ORFs with overlapping verified } \\
\text { genes in parenthesis }\end{array}$} \\
\hline & +++ & YLR235C (TOP3), YOR309C (NOP58), YDR417C (RPL12B) \\
\hline & ++ & $\begin{array}{l}\text { YOLO79W (REX4), YDR433W (NPL3), YDR442W (SSN2), } \\
\text { YJL12OW (RPE1), BUD28 (RPL22A), APQ13 (NET1), YPRO38W } \\
\text { (ERV2), YPRO44C (RPL43A) }\end{array}$ \\
\hline & + & YPL205C (HRR25), YML013C-A (SEL1), YNL140C (RLR1) \\
\hline
\end{tabular}

Some genes are listed more than once, due to being annotated with more than one function. Genes tentatively identified through their overlaps with dubious ORFs have been added in parentheses. The sensitivity to $5-\mathrm{FU}(15 \mu \mathrm{g} / \mathrm{mL})$ was scored from + (least sensitive) to ++++ (complete growth inhibition). 
5-FU sensitivity. Figure 1 shows the various modifications catalyzed by the enzymes found in our screen. Trm1p is responsible for the $\mathrm{m}_{2}^{2} \mathrm{G} 26$ modification (Hopper et al. 1982) while Trm10p catalyzes the formation of $\mathrm{m}^{1} \mathrm{G}$ 9 (Jackman et al. 2003). Trm $8 p$ and Trm $82 p$ form a complex that catalyzes the formation of $\mathrm{m}^{7} \mathrm{G} 46$. It is $\operatorname{Trm} 8 \mathrm{p}$ that contains the catalytic activity (Alexandrov et al. 2005). Mod5p catalyzes the transfer of an isopentenyl group to A37 in the anticodon loop (Dihanich et al. 1987). Puslp catalyzes the formation of pseudouridine at positions $26,27,28,34,35,36,65$, and 67 in tRNA (Motorin et al. 1998), and at position 44 in U2 snRNA (Massenet et al. 1999). Dus3p is required for dihydrouridine modification of U47 in cytoplasmic tRNA (Xing et al. 2004), while Tan1p catalyzes N4-acetylcytidine formation at position 12 in tRNA ${ }^{\text {Ser }}$ (Johansson and Byström 2004). Kti12p and Iki3p/Ekplp, finally, are subunits of the Elongator. This complex was proposed to function in RNA polymerase II elongation (Otero et al. 1999), but most of the Elongator is found in the cytoplasm, where it is involved in wobble uridine methylation of tRNA (Huang et al. 2005; Lu et al. 2005; Jablonowski et al. 2006).

\section{The 5-fluorouracil sensitivity of tRNA modification mutants is enhanced at $38^{\circ} \mathrm{C}$}

Since the tRNA modification mutants were among the most sensitive mutants recovered in our screen, we decided to focus our work on these mutants. A possible mechanism for their increased 5-FU sensitivity is suggested by the fact that 5-FU inhibits several tRNA modifying enzymes, including pseudouridine synthase and tRNA 5-methyluridine

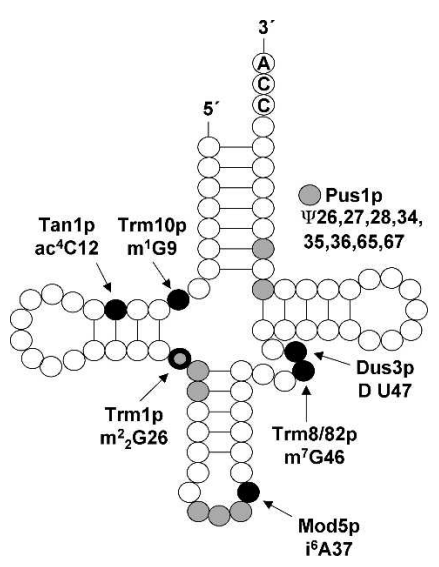

FIGURE 1. Schematic picture of a tRNA molecule with positions that are modified by enzymes that appeared in our screen. N1-methylguanosine $\left[\mathrm{m}^{1} \mathrm{G}_{9}\right]$, N4-acetylcytidine $\left[\mathrm{ac}^{4} \mathrm{C}_{12}\right], \mathrm{N} 2, \mathrm{~N} 2$-methylguanosine $\left[\mathrm{m}_{2}^{2} \mathrm{G}_{26}\right]$, N6-isopentenyladenosine $\left[\mathrm{i}^{6} \mathrm{~A}_{37}\right]$, N7-methylguanosine $\left[\mathrm{m}^{7} \mathrm{G}_{46}\right]$, dihydrouridine $\left[\mathrm{D}_{47}\right]$, and pseudouridine $[\Psi 26-28,34-36$, $65,67]$ modifications are catalyzed by Trm10p, Tan $1 p$, Trm1p, Mod5p, Trm8/82p, Dus3p, and Pus1p, respectively. Pseudouridine is shown in gray, other modifications in black. Position 26 can be modified by either Puslp or Trmlp, depending on whether it is a uridine or a guanosine. methyltransferase (Frendewey et al. 1982; Santi and Hardy 1987; Samuelsson 1991; Huang et al. 1998). The latter enzyme is not essential (Nordlund et al. 2000), but tRNA pseudouridine synthase is, as evidenced by the lethality of a pus1 pus4 double knockout (Grosshans et al. 2001). Accordingly, inhibition of tRNA pseudouridine synthase might contribute to the cytotoxicity of 5-FU. We further note that tRNA modifying enzymes frequently lack mutant phenotypes, particularly those enzymes that modify positions outside the anticodon loop. It was recently shown, however, that two such modifications together can be important for tRNA stability, even if neither modification on its own is essential (Alexandrov et al. 2006).

Based on these findings, we reasoned that loss of one tRNA modification enzyme by a deletion of the encoding gene and loss of others by 5-FU inhibition of their activities might produce a synthetic growth phenotype due to reduced tRNA stability. A testable prediction of this hypothesis is that the effect should be more pronounced at an elevated temperature, where tRNA is more easily destabilized, as in the case of double mutants for tRNA modifying enzymes (Alexandrov et al. 2006). We therefore tested how different single mutants respond to $5-\mathrm{FU}$ at $38^{\circ} \mathrm{C}$. To make it easier to detect an increase in 5-FU sensitivity, we used a low concentration $(1 \mu \mathrm{g} / \mathrm{mL})$. As shown in Figure 2, we found that growth at $38^{\circ} \mathrm{C}$ strongly increases the $5-\mathrm{FU}$ sensitivity of the trm8, trm82, trm10, pus1, and tan 1 mutants. The trm 1 mutant is partially temperature sensitive in the absence of 5-FU, but its growth is completely inhibited by $5-\mathrm{FU}$ at $38^{\circ} \mathrm{C}$. In contrast, only a small effect was seen in the $d u s 3, \bmod 5$, and $l o s 1$ mutants. We also tested 12 5-FUsensitive mutants that affect rRNA processing and four that affect processes unrelated to RNA metabolism. Six of these 16 mutants are partially temperature sensitive and also show a weak additional effect of 5 -FU at $38^{\circ} \mathrm{C}$, but only tom 1 shows complete growth inhibition under these conditions, similar to trm1. Of the remaining eight mutants, only $\operatorname{lsm} 6$ was clearly more sensitive to $5-\mathrm{FU}$ at $38^{\circ} \mathrm{C}$. We conclude that most of the tRNA modification mutants that we tested are highly sensitive to 5 - $\mathrm{FU}$ at $38^{\circ} \mathrm{C}$, while most of the other mutants are not.

\section{Genetic interactions between tRNA-processing mutants at $38^{\circ} \mathrm{C}$ resemble those seen in the presence of 5 -fluorouracil}

We proceeded to test how the tRNA-processing mutants interact genetically, both with respect to 5-FU sensitivity and with respect to sensitivity to other forms of stress. Double mutants were made for all possible combinations of trm1, trm8, trm82, trm10, mod5, pus1, and los1. The 21 double mutants, all of which were viable, were first tested for growth under normal conditions. None of them showed a growth defect when serially diluted onto SC plates and incubated at $30^{\circ} \mathrm{C}$ (data not shown). 


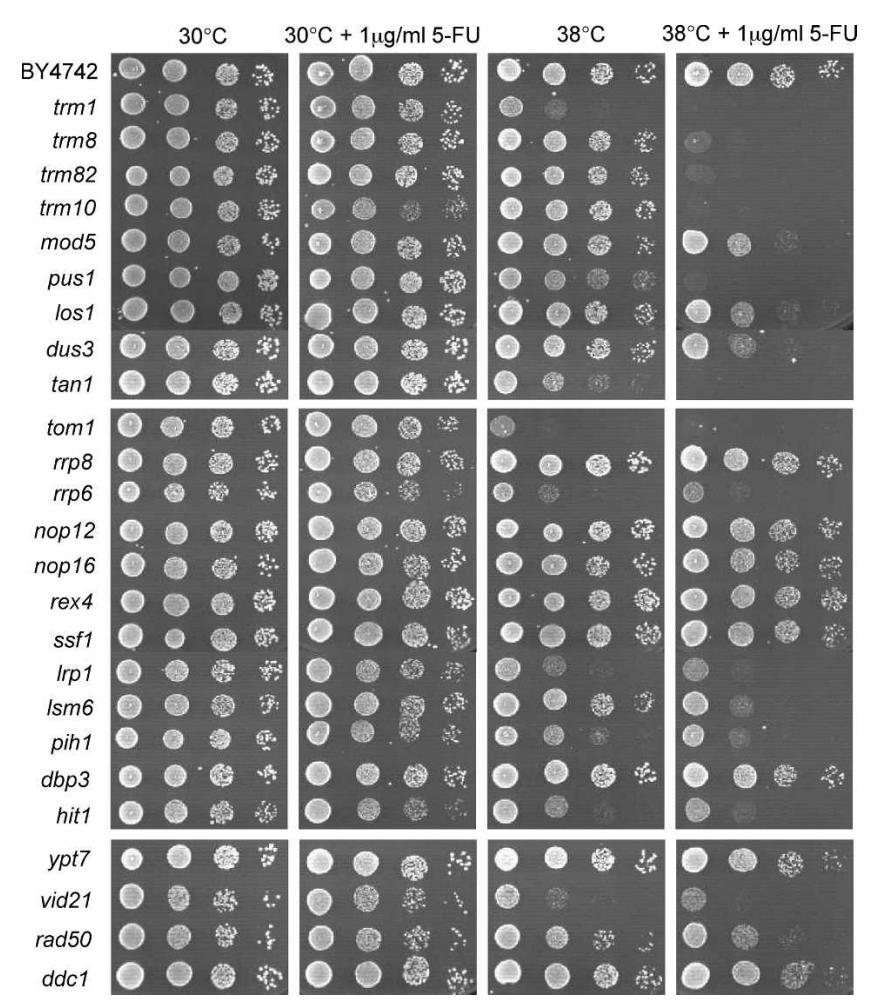

FIGURE 2. Temperature sensitivity of different tRNA modification mutants in the presence of 5-FU. The strains were grown to mid-log phase and spotted in serial 10-fold dilutions onto synthetic complete media in the presence or in the absence of 5-FU $(1 \mu \mathrm{g} / \mathrm{mL})$. The plates were incubated at either $30^{\circ} \mathrm{C}$ or $38^{\circ} \mathrm{C}$ for $2 \mathrm{~d}$. The top panes show the wild type and mutants involved in tRNA modification and maturation, the middle panes mutants involved in rRNA maturation and ribosome biogenesis, and the bottom panes some other 5-FU sensitive mutants that were recovered in the screen.

We proceeded to test all mutants for growth at $38^{\circ} \mathrm{C}$ and in the presence of $5 \mu \mathrm{g} / \mathrm{mL}$ 5-FU. As a control, we also monitored growth at $16^{\circ} \mathrm{C}$ and in the presence of $1 \mathrm{M} \mathrm{NaCl}$, stress conditions that are not expected to affect tRNA stability. None of the single mutants was significantly affected under any of these conditions, except for a complete growth inhibition of the trm 10 mutant on 5-FU (Table 2). At $38^{\circ} \mathrm{C}$, the mutants fall into four distinct groups with respect to their synthetic interactions. First, trm 1 interacts with trm10, $\bmod 5$, pus1, and los 1 , but not with trm8 or trm82. Second, trm8 and trm 82 interact with trm10, pus1, and los1, but not with trm1 or mod5. Third, mod5 interacts only with trm1. Fourth, trm10, pus1, and los1 interact with all other mutations except mod5. Interestingly, a very similar pattern is seen in the presence of $5-\mathrm{FU}$, though the interactions of trm 10 cannot be scored in that case due to the high sensitivity of the trm10 single mutant. The only significant difference is that mod5 also interacts with $\operatorname{trm} 8$, trm82, and los 1 on 5-FU. In contrast, a different pattern is seen in the presence of $1 \mathrm{M} \mathrm{NaCl}$, where trm 1 only interacts with mod5, and los 1 only interacts with trm10 and $\bmod 5$. At $16^{\circ} \mathrm{C}$, finally, we saw only one strong interaction, between trm 1 and los 1 , and one weaker interaction, between trm82 and pus1.

\section{The wild type is temperature sensitive at higher concentrations of 5 -fluorouracil}

The experiments in Figure 2 were carried out at a low concentration of 5-FU to make it possible to detect an increased 5-FU sensitivity at $38^{\circ} \mathrm{C}$ for the already 5-FUsensitive mutants. No effect was therefore seen in the wildtype strain. However, if our hypothesis is correct and 5-FU causes tRNA destabilization by inhibiting tRNA modification, then a higher concentration of 5-FU should cause destabilization also in the wild type. This would also manifest itself as temperature sensitivity in the presence of 5-FU. We therefore proceeded to test how the wild type responds to different concentrations of 5-FU both at $30^{\circ} \mathrm{C}$ and at $38^{\circ} \mathrm{C}$. As shown in Figure 3, the wild type is indeed temperature sensitive in the presence of higher concentrations of 5-FU. Thus, it failed to grow in the presence of $25 \mu \mathrm{g} / \mathrm{mL} 5$-FU at $38^{\circ} \mathrm{C}$ but did grow in the presence of as much as $45 \mu \mathrm{g} / \mathrm{mL} 5$-FU at $30^{\circ} \mathrm{C}$. At 5 and $15 \mu \mathrm{g} / \mathrm{mL}$, there was an $\sim 10$-fold increase in 5 -FU sensitivity at $38^{\circ} \mathrm{C}$, as estimated from serial dilutions. At an even lower concentration no effect was seen. We conclude that the cytotoxic effect of $5-\mathrm{FU}$ is stronger at $38^{\circ} \mathrm{C}$ also in the wild type.

\section{DISCUSSION}

A better understanding of the mechanism of action of 5-FU may help us to develop more efficient methods for its use, including new combination therapies, which could improve cancer treatment. To find out more about the mechanism of action, we have screened a collection of haploid yeast knockout mutants for increased sensitivity or resistance to 5-FU. No resistant mutants were found, but we identified 138 5-FU sensitive mutants representing 132 unique genes. A functional classification reveals a significant overrepresentation of genes involved in RNA processing and maturation. Thus, we found 10 genes involved in nuclear export of RNA and 12 genes involved in rRNA processing. We also found 11 genes encoding ribosomal proteins or proteins involved in ribosome assembly, functions which are tightly linked to that of rRNA. We conclude that RNA processing and maturation are an important in vivo target for 5-FU. This is consistent with previous suggestions that the effect on RNA metabolism is important for the cytotoxic effect of 5-FU (Parker and Cheng 1990; Heimer and Sartorelli 1992; Longley et al. 2003; Fang et al. 2004).

Two previous studies have screened for 5-FU-sensitive mutants in yeast (Giaever et al. 2004; Lum et al. 2004). Both used haploinsuffiency profiling, in which heterozygous diploids are scored for increased sensitivity or resistance 
TABLE 2. Genetic interactions between tRNA modification mutants

\begin{tabular}{|c|c|c|c|c|c|c|c|}
\hline $5-F U$ & WT & trm1 & trm8 & trm82 & trm10 & mod5 & pus1 \\
\hline trm1 & + & & & & & & \\
\hline trm8 & + & + & & & & & \\
\hline trm82 & + & + & + & & & & \\
\hline trm10 & ++++ & ++++ & ++++ & ++++ & & & \\
\hline $\bmod 5$ & + & ++++ & ++ & ++ & ++++ & & \\
\hline pus1 & + & ++++ & ++ & ++ & ++++ & + & \\
\hline $\operatorname{los} 1$ & & ++++ & ++ & ++ & ++++ & ++ & ++++ \\
\hline $38^{\circ} \mathrm{C}$ & WT & trm1 & trm8 & trm82 & $\operatorname{trm} 10$ & mod5 & pus1 \\
\hline$\overline{t r m 1}$ & + & & & & & & \\
\hline trm8 & & + & & & & & \\
\hline trm82 & & $(+)$ & & & & & \\
\hline $\operatorname{trm} 10$ & & +++ & ++++ & ++ & & & \\
\hline mod5 & & ++++ & & & & & \\
\hline pus1 & + & ++ & ++++ & ++++ & ++++ & + & \\
\hline los 1 & & ++++ & ++++ & ++++ & +++ & & ++++ \\
\hline $16^{\circ} \mathrm{C}$ & WT & trm1 & trm8 & trm82 & trm10 & $\bmod 5$ & pus1 \\
\hline trm1 & & & & & & & \\
\hline trm8 & & & & & & & \\
\hline trm82 & & & & & & & \\
\hline $\operatorname{trm} 10$ & & & & & & & \\
\hline $\bmod 5$ & & & & & & & \\
\hline pus1 & & & $(+)$ & ++ & & & \\
\hline $\operatorname{los} 1$ & & ++++ & $(+)$ & $(+)$ & $(+)$ & $(+)$ & \\
\hline $\mathrm{NaCl}$ & WT & trm1 & trm8 & trm82 & $\operatorname{trm} 10$ & $\bmod 5$ & pus1 \\
\hline trm1 & + & & & & & & \\
\hline trm8 & & + & & & & & \\
\hline trm82 & & + & & & & & \\
\hline $\operatorname{trm} 10$ & & + & ++ & + & & & \\
\hline $\bmod 5$ & + & ++ & ++ & ++ & + & & \\
\hline pus1 & & + & +++ & +++ & +++ & + & \\
\hline $\operatorname{los} 1$ & & + & + & + & ++ & ++ & + \\
\hline
\end{tabular}

The table shows growth inhibition of single mutants (column 1 ) and double mutants (columns 2-7) under different conditions. The scores range from + (least sensitive) to ++++ (complete growth inhibition). No mark means that the strain was as sensitive as the wild type. Double mutants that show a significant growth inhibition (at least ++ ) are marked with dark gray. The trm10 double mutants are marked with light gray on 5-FU since the complete growth inhibition of the trm10 single mutant made them non-informative for genetic interactions on 5 -FU. The concentration of $5-\mathrm{FU}$ was $5 \mu \mathrm{g} / \mathrm{mL}$.

to a drug. Haploinsuffiency profiling differs significantly from screening of haploid mutants (Deutschbauer et al. 2005). For example, it includes essential genes, which are lethal in the haploid. On the other hand, it will miss all genes for which $50 \%$ of the wild-type gene product is adequate for full in vivo function. It is therefore interesting to compare the results of our screen and the two haploinsuffiency screens. Most of the genes found in the latter are involved in rRNA processing. Five genes were found in both screens, which is a significant overlap given the small number of genes (19 and nine). Our screen shows a very different profile. Thus, only four of our 132 unique genes (SSF1, NOP58, RLR1, and RRP6) appeared in one of the two haploinsuffiency screens. Furthermore, while rrp6 was the most sensitive mutant found by Lum et al. (2004), it was only moderately sensitive in our screen. Our most sensitive mutant, trm10, is not known to be involved in rRNA processing but in tRNA modification, as were several other highly sensitive mutants. This highlights tRNA modification as an important in vivo target of 5-FU, and we therefore focused our studies on this process.

Modifications of tRNA are found in all living organisms, and the modifying enzymes are highly conserved, suggesting that they are important (Hopper and Phizicky 2003). Paradoxically, however, knockouts of these enzymes frequently lack obvious phenotypes, particularly if they modify bases outside the anticodon loop. A clue to the role of these nonessential tRNA modifications has emerged from the work of Alexandrov et al. (2006). They found that, while knockouts of TRM8 or TRM82 encoding the $\mathrm{m}^{7} \mathrm{G} 46$ methyltransferase lack phenotypes, double knockouts of either TRM8 or TRM82 and any of seven other genes encoding nonessential tRNA modification enzymes are temperature sensitive. They further showed that the temperature-sensitive phenotype of one double mutant, trm 8 trm4, is due to rapid decay of tRNA ${ }^{\mathrm{Val}(\mathrm{AAC})}$. Thus, two tRNA modifications together can play an important role for tRNA stability, even if neither modification on its own is essential (Alexandrov et al. 2006).

Interestingly, 5-FU inhibits several tRNA modifying enzymes by forming covalent complexes between the enzyme and 5-FU-substituted tRNA. Pseudouridine synthase and tRNA 5-methyluridine methyltransferase are both inhibited (Frendewey et al. 1982; Santi and Hardy 1987; Samuelsson 1991; Huang et al. 1998), and it is likely that other tRNA modifying enzymes may be similarly affected. Furthermore, Johnson et al. (1980) have shown that the total amounts of pseudouridine, thymidine, and H-5,6uridine in mature tRNAs are significantly reduced in yeast cells treated with as little as $1 \mu \mathrm{g} / \mathrm{mL} 5$-FU, beyond what can be accounted for by stoichiometric 5-FU incorporation. This suggests the possibility that loss of one tRNA modifying enzyme by deletion of its gene, and loss of others by 5-FU inhibition, together might reduce some tRNA modification(s) below a critical level, and thus produce a synthetic phenotype similar to that seen by Alexandrov et al. (2006) for the trm8 trm4 double mutant (Fig. 4). One prediction of this hypothesis is that growth inhibition by 5 -FU should be more pronounced at $38^{\circ} \mathrm{C}$, where tRNA is more easily destabilized. Another prediction is that the effect should be confined to mutants that affect nonessential modifications outside the anticodon loop, which are important for tRNA stability (Alexandrov et al. 2006). In contrast, mutants affecting modifications within the anticodon loop, tRNA export, rRNA processing, 


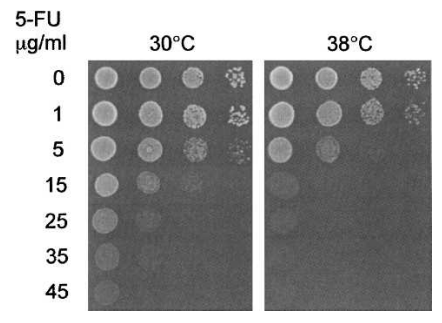

FIGURE 3. Temperature sensitivity of wild-type cells in the presence of higher concentrations of 5-FU. The strains were grown to mid-log phase and spotted in serial 10-fold dilutions onto synthetic complete media in the presence or in the absence of different concentrations of $5-\mathrm{FU}$, as indicated in the figure. The plates were incubated at either $30^{\circ} \mathrm{C}$ or $38^{\circ} \mathrm{C}$ for $2 \mathrm{~d}$.

or other cellular functions should not be temperature sensitive on 5-FU.

Our experiments confirmed both predictions (Fig. 2). Thus, we found that the trm1, trm8, trm82, trm10, pus1, and $\tan 1$ mutants, all of which affect tRNA modifications outside the anticodon loop, show increased sensitivity to 5 -FU at $38^{\circ} \mathrm{C}$. Only one such mutant, $d u s 3$, did not. In contrast, the mod5 mutant, which affects a modification in the anticodon loop, and los 1 , which affects pre-tRNA export and splicing, were not significantly more sensitive to $5-\mathrm{FU}$ at $38^{\circ} \mathrm{C}$. Of the 165 -FU-sensitive mutants that affect other cellular processes, only tom 1 and 1 sm 6 showed a clearly increased sensitivity to $5-\mathrm{FU}$ at $38^{\circ} \mathrm{C}$. It should be noted that Tom $1 \mathrm{p}$ and Lsm6p affect many processes, including transcription and mRNA turnover. They may therefore also have indirect effects on tRNA modification. Lsm6p has in fact also been implicated in tRNA processing (Kufel et al. 2003).

The notion that the 5-FU sensitivity of the tRNA modification mutants involves tRNA destabilization is also supported by our results obtained with double mutants (Table 2). Thus, we found that the pattern of synthetic interactions between different mutants in the presence of 5 -FU resembles those seen at $38^{\circ} \mathrm{C}$. In contrast, different patterns of synthetic interactions are seen at $16^{\circ} \mathrm{C}$ and in the presence of $1 \mathrm{M} \mathrm{NaCl}$, stress conditions that are not expected to affect tRNA stability (Table 2). This makes sense if sensitivity to 5-FU and to a higher temperature both involve destabilization of tRNA. The 5-FU-sensitive target(s) that mediate this effect remain to be identified. One likely candidate is pseudouridine synthase, for several reasons. First, tRNA pseudouridine synthases are known to be inhibited by 5-FU (Frendewey et al. 1982; Samuelsson 1991; Huang et al. 1998). Second, pseudouridylation is the most common tRNA modification, which affects multiple sites in each tRNA (Fig. 1). Third, pseudouridylation is essential, as shown by the lethality of a pus 1 pus 4 double mutant (Grosshans et al. 2001). Fourth, pseudouridine formation in yeast tRNA was particularly sensitive to 5-FU (Johnson et al. 1980). That pseudouridylation is an impor- tant in vivo target for 5-FU is also supported by the finding that 5-FU inhibits pre-mRNA splicing in human cells, by blocking pseudouridylation of U2 snRNA (Zhao and Yu 2007). However, inhibition of other 5-FU-sensitive tRNA modifying enzymes such as 5-methyluridine methyltransferase (Frendewey et al. 1982; Santi and Hardy 1987) could contribute to the cytotoxic effect of 5-FU.

Alexandrov et al. (2006) found that destabilization by the trm8 trm4 double mutant was specific for certain tRNAs, in particular tRNA ${ }^{\mathrm{Val}(\mathrm{AAC})}$, and also that the latter was rapidly degraded after a temperature shift. It remains to be seen if the temperature-sensitive phenotype of our single mutants in the presence of 5-FU also is due to a specific effect on certain tRNAs, or if it reflects a general effect on tRNA stability. Preliminary experiments did not reveal a generally increased tRNA degradation under restrictive conditions (data not shown). However, growth inhibition would only require tRNA destabilization, not degradation, and, if components of the degradation pathway are sensitive to 5-FU, the latter process might be delayed in the presence of the drug. Nor can a more specific effect on certain tRNAs be excluded. Further experiments are needed to answer these questions.

Significantly, we found that also the wild-type strain is temperature sensitive in the presence of higher concentrations of 5-FU (Fig. 3). This suggests that tRNA destabilization contributes to the cytoxic effect of 5-FU in wild-type cells,
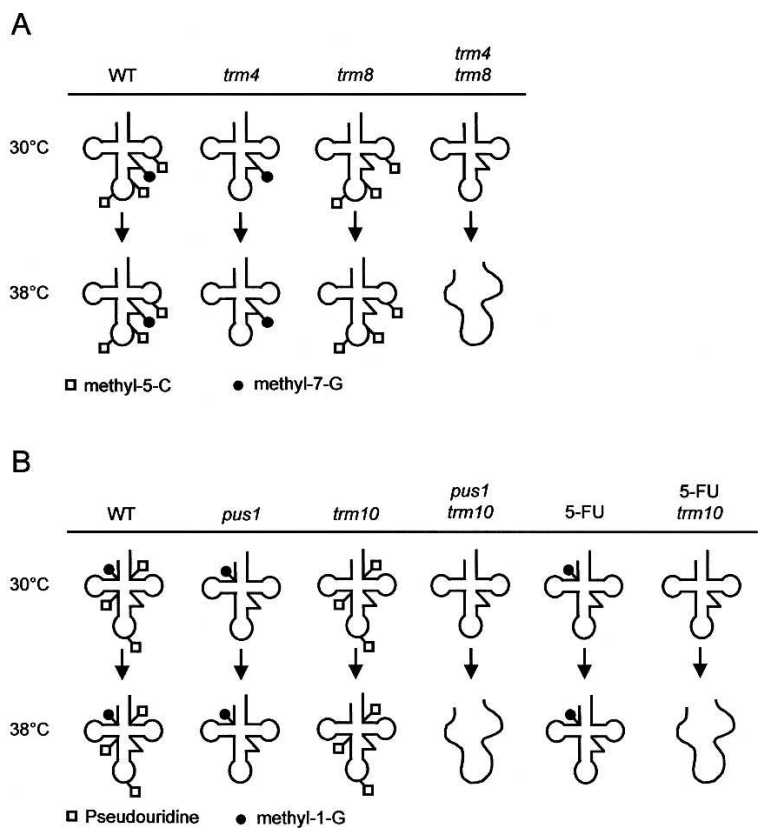

FIGURE 4. Model for how loss of tRNA modifications causes tRNA destabilization, which is detected as temperature sensitivity. (A) Synthetic interaction between the trm 4 and trm 8 mutations, as described by Alexandrov et al. (2006). (B) Synthetic interaction between the pus 1 and trm10 mutations, or alternatively between 5-FU treatment (which inhibits Puslp) and the trm10 mutation, as described in the present paper. Destabilized (but not necessarily degraded) tRNA is shown as a melted loop. 
and not just in tRNA modification mutants. Interestingly, results obtained in mammalian cells (Kido et al. 1991) and one clinical trial (Kouloulias et al. 2005) suggest that hyperthermia can potentiate the effect of 5-FU. Our results provide a possible explanation for this phenomenon and suggest that hyperthermia should be explored further as a possible way to enhance the efficiency of 5-FU in cancer treatment.

\section{MATERIALS AND METHODS}

\section{Yeast strains and growth media}

Yeast deletion strains in the BY4742 background, where the ORF in each deletion strain has been replaced by a KanMX4 cassette, were obtained from the Euroscarf collection (http://www.unifrankfurt.de/fb15/mikro/euroscarf). MAT $\alpha$ deletion strains were used in the screen, and congenic MATa strains were used to verify the results. Double deletion strains were made by crosses between these strains followed by tetrad dissection and are listed in Table 3. Rich (YPD) and synthetic complete (SC) media were prepared as described by Sherman et al. (1986) but with twice the normal amount of leucine in the SC media. 5-FU dissolved in $50 \mathrm{mg} / \mathrm{mL}$ glucose was obtained from Apoteksbolaget.

\section{Screen for 5-FU sensitivity and phenotypic analysis}

Yeast strains were grown on YPD plates, suspended in sterile water to a density of $3 \times 10^{6}$ cells $/ \mathrm{mL}$, and then sequentially diluted in water using 20 -fold steps. One microliter of each dilution was

TABLE 3. Yeast strains

\begin{tabular}{|c|c|}
\hline Strain & Relevant genotype \\
\hline H1517 & 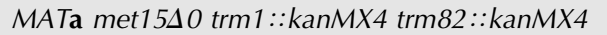 \\
\hline H1518 & MATa lys $2 \Delta 0 \operatorname{trm} 1:: \operatorname{kanMX} 4 \bmod 5:: \operatorname{kanM} X 4$ \\
\hline $\mathrm{H} 1520$ & 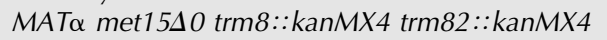 \\
\hline $\mathrm{H} 1521$ & 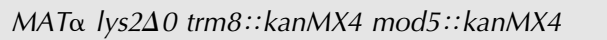 \\
\hline $\mathrm{H} 1522$ & MATa lys $2 \Delta 0$ trm8::kanMX4 pus1::kanMX4 \\
\hline H1523 & 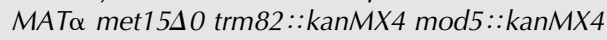 \\
\hline H1524 & 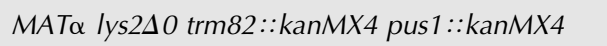 \\
\hline H1525 & MATa lys $2 \Delta 0$ mod5::kanMX4 pus1::kanMX4 \\
\hline H1526 & 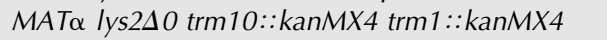 \\
\hline H1527 & 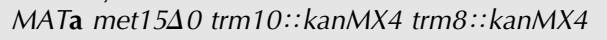 \\
\hline H1528 & 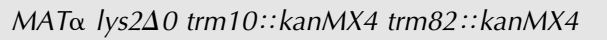 \\
\hline H1529 & 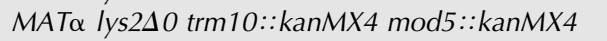 \\
\hline $\mathrm{H} 1531$ & 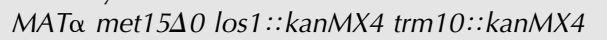 \\
\hline $\mathrm{H} 1532$ & 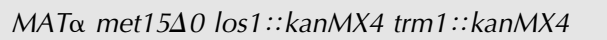 \\
\hline H1533 & 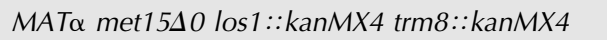 \\
\hline $\mathrm{H} 1534$ & MATa lys2 200 los $1::$ kanMX4 trm82::kanMX4 \\
\hline $\mathrm{H} 1535$ & MATa lys $2 \Delta 0 \operatorname{los} 1:: \operatorname{kanMX} 4 \bmod 5:: \operatorname{kanMX} 4$ \\
\hline H1536 & 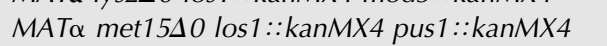 \\
\hline $\mathrm{H} 1600$ & MATa lys $2 \Delta 0 \operatorname{trm} 1:: \operatorname{kanMX} 4 \operatorname{trm} 8:: \operatorname{kanMX} 4$ \\
\hline $\mathrm{H} 1601$ & 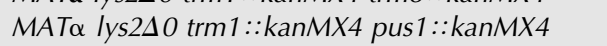 \\
\hline H1602 & 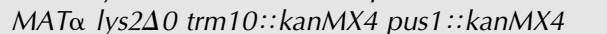 \\
\hline
\end{tabular}

All strains also carry the his $3 \Delta 1$ leu2 $\Delta O$ ura3 $\Delta O$ markers from the BY4742 background. spotted onto SC plates containing $15 \mu \mathrm{g} / \mathrm{mL} 5-\mathrm{FU}$, using a Quadra 96 SV model 250 pipetting robot. Plates were incubated at $30^{\circ} \mathrm{C}$ and growth was monitored after two days. Sensitive mutants were retested using both MAT $\alpha$ and MATa deletion strains. Liquid yeast cultures were grown overnight at $30^{\circ} \mathrm{C}$ in SC. The precultures were diluted into fresh media to a final $\mathrm{OD}_{600}$ of 0.1 and grown to mid-log phase. The cells were harvested, and three serial 10 -fold dilutions in water were prepared. A $5 \mu \mathrm{L}$ aliquot of each dilution was spotted onto SC control plates and SC plates containing $15 \mu \mathrm{g} / \mathrm{mL}$ 5-FU. Growth was monitored after two days at $30^{\circ} \mathrm{C}$. Mutant strains were also tested on YPG and YPE to score petite phenotypes. Strains that showed a reduced growth on the SC control plates, including petite strains, were not studied further since a reduced growth makes it more difficult to score drug sensitivity. Phenotyping under other conditions used the same serial dilution procedure.

\section{ACKNOWLEDGMENTS}

We thank Drs. Eva Murén and Kerstin Stråby for helpful advice. This work was supported by grants from the Swedish Cancer Society, the Swedish Research School in Genomics and Bioinformatics, and the Swedish Foundation for Strategic Research.

Received December 19, 2007; accepted January 17, 2008.

\section{REFERENCES}

Alexandrov, A., Grayhack, E.J., and Phizicky, E.M. 2005. tRNA m ${ }^{7} \mathrm{G}$ methyltransferase Trm8p/Trm82p: Evidence linking activity to growth phenotype and implicating Trm82p in maintaining levels of active Trm8p. RNA 11: 821-830.

Alexandrov, A., Chernyakov, I., Gu, W., Hiley, S.L., Hughes, T.R., Grayhack, E.J., and Phizicky, E.M. 2006. Rapid tRNA decay can result from lack of nonessential modifications. Mol. Cell 21: $144-145$.

Antonelli-Bousquet, C., Vanrobays, E., Gelugne, J.-P., CaizerguesFerrer, M., and Henry, Y. 2000. Rrp8 is a yeast nucleolar protein functionally linked to Garlp and involved in pre-rRNA cleavage at site A2. RNA 6: 826-843.

Benko, A.L., Vaduva, G., Martin, N.C., and Hopper, A.K. 2000. Competition between a sterol biosynthetic enzyme and tRNA modification in addition to changes in the protein synthesis machinery causes altered nonsense suppression. Proc. Natl. Acad. Sci. 97: 61-66.

Deutschbauer, A.M., Jaramillo, D.F., Proctor, M., Kumm, J., Hillenmeyer, M.E., Davis, R.W., Nislow, C., and Giaever, G. 2005. Mechanisms of haploinsufficiency revealed by genome-wide profiling in yeast. Genetics 169: 1915-1925.

Dihanich, M.E., Najarian, D., Clark, R., Gillman, E.C., Martin, N.C., and Hopper, A.K. 1987. Isolation and characterization of MOD5, a gene required for isopentenylation of cytoplasmic and mitochondrial tRNAs of Saccharomyces cerevisiae. Mol. Cell. Biol. 7: $177-184$.

Duncan, K., Umen, J.G., and Guthrie, C. 2000. A putative ubiquitin ligase required for efficient mRNA export differentially affects hnRNP transport. Curr. Biol. 10: 687-696.

Fang, F., Hoskins, J., and Butler, J.S. 2004. 5-Fluorouracil enhances exosome-dependent accumulation of polyadenylated rRNAs. Mol. Cell. Biol. 24: 10766-10776.

Frendewey, D.A., Kladianos, D.M., Moore, V.G., and Kaiser, I.I. 1982. Loss of tRNA 5-methyluridine methyltransferases and pseudouridine 
synthase activities in 5-fluorouracil and 1-(tetrahydro-2-furanyl)5-fluorouracil (ftorafur)-treated Escherichia coli. Biochim. Biophys. Acta 697: 31-40.

Giaever, G., Flaherty, P., Kumm, J., Proctor, M., Nislow, C., Jaramillo, D.F., Chu, A.M., Jordan, M.I., Arkin, A.P., and Davis, R.W. 2004. Chemogenomic profiling: Identifying the functional interactions of small molecules in yeast. Proc. Natl. Acad. Sci. 101: 793-798.

Grosshans, H., Lecointe, F., Grosjean, H., Hurt, E., and Simos, G. 2001. Puslp-dependent tRNA pseudouridinylation becomes essential when tRNA biogenesis is compromised in yeast. J. Biol. Chem. 276: 46333-46339.

Heimer, R. and Sartorelli, A.C. 1992. RNA-directed actions of 5fluorouridine in hemin stimulated K-562 erythroleukemia cells. Cancer Biochem. Biophys. 12: 221-239.

Hopper, A.K. and Phizicky, E.M. 2003. tRNA transfers to the limelight. Genes \& Dev. 17: 162-180.

Hopper, A.K., Furukawa, A.H., Pham, H.D., and Martin, N.C. 1982. Defects in modification of cytoplasmic and mitochondrial transfer RNAs are caused by single nuclear mutations. Cell 28: 543550.

Huang, L., Pookanjanatavip, M., Gu, X., and Santi, D.V. 1998. A conserved aspartate of tRNA pseudouridine synthase is essential for activity and a probable nucleophilic catalyst. Biochemistry 37: 344-351.

Huang, B., Johanson, M.J., and Bystrom, A.S. 2005. An early step in wobble uridine tRNA modification requires the Elongator complex. RNA 11: 424-436.

Ito, T., Chiba, T., Ozawa, R., Yoshida, M., Hattori, M., and Sakaki, Y. 2001. A comprehensive two-hybrid analysis to explore the yeast protein interactome. Proc. Natl. Acad. Sci. 98: 4569-4574.

Jablonowski, D., Zink, S., Mehlgarten, C., Daum, G., and Schaffrath, R. 2006. tRNA Glu wobble uridine methylation by Trm9 identifies Elongator's key role for zymocin-induced cell death in yeast. Mol. Microbiol. 59: 677-688.

Jackman, J.E., Montage, R.K., Melik, H.S., and Phizicky, E.M. 2003. Identification of the yeast gene encoding the tRNA $\mathrm{m}^{1} \mathrm{G}$ methyltransferases responsible for modification at position 9. RNA 9: $574-585$.

Johansson, M.J.O. and Byström, A.S. 2004. The Saccharomyces cerevisiae TAN1 gene is required for $N^{4}$-acetylcytidine formation in tRNA. RNA 10: 712-719.

Johnson, J.D., Kaiser, I.I., and Horowitz, J. 1980. Effects of 5fluorouracil on the formation of modified nucleosides in yeast transfer RNA. Biochim. Biophys. Acta 607: 285-294.

Kido, Y., Kuwano, H., Maehara, Y., Mori, M., Matsuoka, H., and Sugimachi, K. 1991. Increased cytotoxicity of low-dose, longduration exposure to 5-fluorouracil of V-79 cells with hyperthermia. Cancer Chemother. Pharmacol. 28: 251-254.

Kouloulias, V., Plataniotis, G., Kouvaris, J., Dardoufas, C., Gennatas, C., Uzunoglu, N., Papavasiliou, C., and Vlahos, L. 2005. Chemoradiotherapy combined with intracavitary hyperthermia for anal cancer: Feasibility and long-term results from a phase II randomized trial. Am. J. Clin. Oncol. 28: 91-99.

Kufel, J., Allmang, E., Beggs, J., and Tollervey, D. 2003. Lsm proteins are required for normal processing and stability of ribosomal RNAs. J. Biol. Chem. 278: 2147-2156.
Longley, D.B., Harkin, D.P., and Johnston, P.G. 2003. 5-fluorouracil: Mechanism of action and clinical strategies. Nat. Rev. Cancer 3: 330-338.

Lu, J., Huang, B., Esberg, A., Johanson, M.J., and Byström, A.S. 2005. The Kluyveromyces lactis $\gamma$-toxin targets tRNA anticodons. RNA 11: $1648-1654$.

Lum, P.Y., Armour, C.D., Stepaniants, S.B., Cavet, G., Wolf, M.K., Butler, J.S., Hinshaw, J.C., Garnier, P., Prestwich, G.D., Leonardson, A., et al. 2004. Discovering modes of action for therapeutic compounds using a genome-wide screen of yeast heterozygotes. Cell 116: 121-137.

Massenet, S., Motorin, Y., Lafontaine, D.L.J., Hurt, E.C., Grosjean, H., and Branlant, C. 1999. Pseudouridine mapping in the Saccharomyces cerevisiae spliceosomal U small nuclear RNAs (snRNAs) reveals that pseudouridine synthase Puslp exhibits a dual substrate specificity for U2 snRNA and tRNA. Mol. Cell. Biol. 19: 2142-2154.

Motorin, Y., Keith, G., Simon, C., Foiret, D., Simos, G., Hurt, E., and Grosjean, H. 1998. The yeast tRNA:pseudouridine synthase Puslp displays a multisite substrate specificity. RNA 4: 856-869.

Murén, E., Oyen, M., Barmark, G., and Ronne, H. 2001. Identification of yeast deletion strains that are hypersensitive to brefeldin A or monensin, two drugs that affect intracellular transport. Yeast 18: 163-172.

Nordlund, M.E., Johansson, J.O., von Pawel-Rammingen, U., and Bystrom, A.S. 2000. Identification of the TRM2 gene encoding the $\operatorname{tRNA}\left(\mathrm{m}^{5} \mathrm{U}_{54}\right)$ methyltransferase of Saccharomyces cerevisiae. RNA 6: $844-860$.

Otero, G., Fellows, J., Li, Y., de Bizemont, T., Dirac, A.M., Gustafsson, C.M., Erdjument-Bromage, H., Tempst, P., and Svejstrup, J.Q. 1999. Elongator, a multisubunit component of a novel RNA polymerase II holoenzyme for transcriptional elongation. Mol. Cell 3: 109-118.

Parker, W.B. and Cheng, Y.C. 1990. Metabolism and mechanism of action of 5-fluorouracil. Pharmacol. Ther. 48: 381-395.

Saleh, A., Collart, M., Martens, J.A., Genereaux, J., Allard, S., Cote, J., and Brandl, C.J. 1998. TOM1p, a yeast hect-domain protein which mediates transcriptional regulation through the ADA/SAGA coactivator complexes. J. Mol. Biol. 282: 933-946.

Samuelsson, T. 1991. Interaction of transfer RNA pseudouridine synthases with RNAs substituted with fluorouracil. Nucleic Acids Res. 19: 6139-6144. doi: 10.1093/nar/19.22.6139.

Santi, D.V. and Hardy, L.W. 1987. Catalytic mechanism and inhibition of tRNA (uracil-5-)methyltransferase: Evidence for covalent catalysis. Biochemistry 26: 8599-8606.

Sherman, F., Fink, G., and Hicks, J.B 1986. Methods in yeast genetics. A laboratory manual. Cold Spring Harbor Laboratory, Cold Spring Harbor, NY.

Tabb, A.L., Utsugi, T., Wooten-Kee, C.R., Sasaki, T., Edling, S.A., Gump, W., Kikuchi, Y., and Ellis, S.R. 2001. Genes encoding ribosomal proteins Rps0A/B of Saccharomyces cerevisiae interact with TOM1 mutants defective in ribosome synthesis. Genetics 157: 1107-1116.

Xing, F., Hiley, S.L., Hughes, T.R., and Phizicky, E.M. 2004. The specificities of four yeast dihydrouridine synthase for cytoplasmic tRNAs. J. Biol. Chem. 279: 17850-17860.

Zhao, X. and Yu, Y.-T. 2007. Incorporation of 5-fluorouracil into U2 snRNA blocks pseudouridylation and pre-mRNA splicing in vivo. Nucleic Acids Res. 35: 550-558. doi: 10.1093/nar/gkl1084. 

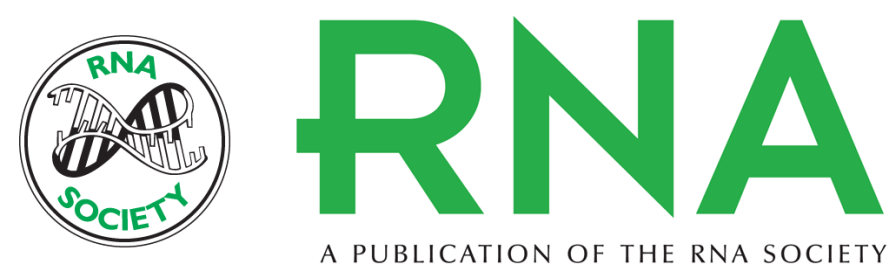

A PUBLICATION OF THE RNA SOCIETY

\section{Evidence that tRNA modifying enzymes are important in vivo targets for 5-fluorouracil in yeast}

Marie Gustavsson and Hans Ronne

RNA 2008 14: 666-674

References This article cites 39 articles, 20 of which can be accessed free at:

http://rnajournal.cshlp.org/content/14/4/666.full.html\#ref-list-1

\section{License}

Email Alerting Receive free email alerts when new articles cite this article - sign up in the box at the Service top right corner of the article or click here. 